\title{
Mismatch excels when ampicillin runs low
}

c6

\section{mutations}

generated

by Pol IV are

retained in

the genome

because MutS

is depleted
Mounting evidence indicates that subinhibitory antibiotic concentrations, which bacteria frequently encounter, can lead to the emergence of de novo resistance mutations. However, the molecular basis of this phenomenon is poorly understood. Matic and colleagues now reveal that low concentrations of $\beta$-lactam antibiotics induce mutagenesis by an RpoS-mediated reduction in replication fidelity.

The stress response $\sigma$-factor RpoS (also known as $\sigma^{\mathrm{S}}$ ) regulates mutagenesis in Escherichia coli by inducing expression of the errorprone DNA polymerase IV (Pol IV), encoded by $\operatorname{din} B$. Because Pol IV has previously been shown to incorporate mutagenic oxidized nucleotides, which are generated during antibiotic exposure, it has been suggested that induction of this polymerase

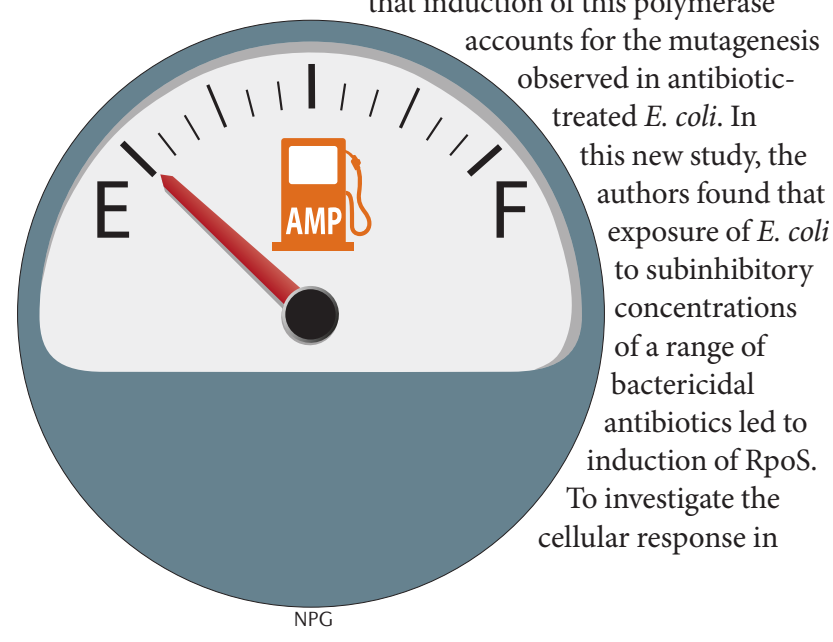

more detail, the $\beta$-lactam antibiotic ampicillin was chosen for further study. Exposure to half the minimum inhibitory concentration of ampicillin resulted in an approximate two-fold increase in RpoS protein levels. The elevated RpoS levels increased mutagenesis by up to eight-fold in ampicillin-treated cells compared with untreated cells. This increased mutation frequency was further shown to depend on Pol IV, as it was abolished by deletion of $\operatorname{din} B$. Together, these data show that subinhibitory antibiotic concentrations increase the expression of RpoS, which is required for Pol IV-mediated mutagenesis.

A detailed evaluation of the mutations generated by ampicillin treatment revealed a bias in $\mathrm{GC} \rightarrow \mathrm{AT}$ and $\mathrm{AT} \rightarrow \mathrm{GC}$ transitions, a typical hallmark of defective mismatch repair. Thus, the authors assessed the involvement of MutS and MutL (which have a role in the repair of replication errors) in mutagenesis. MutS levels were reduced in ampicillin-treated cells, and overexpression of MutS strongly inhibited ampicillin-induced mutagenesis, suggesting that mutations generated by Pol IV are retained in the genome because MutS is depleted.

But what causes the MutS deficiency? Using RNA footprinting assays, the authors found that the small RNA SdsR, which was recently shown to be positively regulated by RpoS, binds to a complementary region in the mutS mRNA, leading to RNase III-mediated cleavage of the resultant duplex. Furthermore, inactivation of the $s d s R$ gene resulted in a substantial reduction in ampicillininduced mutagenesis. Collectively, these data suggest that RpoS induction of SdsR leads to depletion of MutS and a consequent reduction in replication fidelity.

Finally, the authors showed that the RpoS, Pol IV and MutSdepletion pathway is also required for subinhibitory $\beta$-lactam-induced mutagenesis in Vibrio cholerae and Pseudomonas aeruginosa, demonstrating that this is a general phenomenon among bacteria. This study highlights the relevance of subinhibitory antibiotic exposure for the generation of mutations, which in turn increases the probability of acquiring resistance mutations, and these findings therefore have important implications for antibiotic treatment regimens.

Christina Tobin Kåhrström

ORIGINAL RESEARCH PAPER Gutierrez, A. et al. $\beta$-lactam antibiotics promote bacterial mutagenesis via an RpoS-mediated reduction in replication fidelity. Nature Commun. 19 Mar 2013 (doi:10.1038/ncomms2607) 